\title{
Role of aortopexy in the management of primary tracheomalacia and tracheobronchomalacia
}

\author{
P S Malone, E M Kiely
}

\begin{abstract}
Between 1986 and 1988 aortopexy was carried out on 12 patients with primary tracheomalacia and five with tracheobronchomalacia; their median age was 5 months (1 month - 7 years). All patients had stridor, 14 had had cyanotic and apnoeic episodes, and 10 had had 'death' attacks. Eight patients (47\%) had associated gastro-oesophageal reflux. Six patients $(35 \%)$ were helped by aortopexy alone, and a further five (29\%) improved with a combination of aortopexy and antireflux treatment. Four patients required tracheostomy, one of whom died. Aortopexy failed if gastro-oesophageal reflux was present. We recommend aggressive treatment of reflux combined with aortopexy in patients with primary tracheomalacia and tracheobronchomalacia.
\end{abstract}

Tracheomalacia is characterised by an abnormally soft and easily collapsible trachea. It often occurs in association with oesophageal atresia and tracheo-oesophageal fistula, but there is a less common primary form in which the bronchi as well as the trachea may be affected. It causes airway obstruction, particularly at times of high air flow such as when coughing, crying, and feeding. The typical symptom is stridor, which inevitably has an expiratory component. Severe forms produce repeated apnoeic and cyanotic episodes that can progress to 'death' attacks when bradycardia supervenes. Mild cases usually resolve spontaneously at about 1 year of age. In the more severe forms treatment is required to save life.

In the past, treatment consisted of long term intubation and ventilation or tracheostomy, but recently aortopexy has gained favour. Aortopexy entails suturing the anterior wall of the ascending aorta to the posterior surface of the sternum, which in turn pulls the anterior wall of the trachea forward and prevents collapse. Its initial success in the treatment of tracheomalacia associated with oesophageal atresia has encouraged surgeons to use it for the more uncommon primary tracheomalacia and tracheobronchomalacia. ${ }^{1-3}$ To our knowledge there has been no previous study reporting the results of aortopexy in primary tracheomalacia, but the results in tracheobronchomalacia are disappointing. ${ }^{4}$ In 1987 we reported the results of aortopexy in 22 patients with oesophageal atresia. ${ }^{3}$ Using these as historical controls, we analysed our results of aortopexy in the management of primary tracheomalacia and tracheobronchomalacia.

\section{Patients and methods}

The records of all patients with a diagnosis of primary tracheomalacia or tracheobronchomalacia who underwent aortopexy between 1986 and 1988 were reviewed retrospectively. All patients were referred either from the respiratory or ear, nose, and throat departments of the hospital. In each case symptoms were typical of upper airway obstruction with nothing to suggest primary pulmonary disease. Pulmonary function studies were not carried out routinely.

There were 17 patients: 13 boys and four girls. Ages ranged from 1 month-7 years (median 5 months); symptoms are listed in table 1. 'Death' attacks are defined as the development of bradycardia, pallor, and floppiness after a prolonged apnoeic or cyanotic episode. Five patients with death attacks required full cardiopulmonary resuscitation and admission to an intensive care unit. The remaining five patients were given mouth to mouth resuscitation, and one required emergency tracheostomy on admission to hospital. Prolonged hypoxia secondary to a death attack was the only aetiological factor in both patients with cerebral palsy. All patients presenting with apnoeic or cyanotic episodes had further attacks in hospital before operation. Thirteen patients were symptomatic within the neonatal period, seven having had problems since birth. Three developed symptoms between 1 and 6 months of age, and one patient was over 6 months old. Associated features are listed in table 2 . The patient who presented at 7 years of age had had an emergency tracheostomy performed at another unit when she was 3 years old. Numerous

Table 1 Symptoms in 12 patients with tracheomalacia and five with tracheobronchomalacia

\begin{tabular}{lc}
\hline Symptoms & No of patients \\
\hline Stridor & 17 \\
Cyanotic or apnoeic attacks & 14 \\
'Death' attacks & 10 \\
Recurrent respiratory infections & 3
\end{tabular}

Table 2 Associated features in 12 patients with tracheomalacia and five with tracheobronchomalacia

\begin{tabular}{ll}
\hline Feature & $\begin{array}{l}\text { No of } \\
\text { patients }\end{array}$ \\
\hline Gastro-oesophageal reflux 35 weeks' gestation) & $8^{*}$ \\
Prematurity (28, 32, and & 3 \\
Cerebral palsy & 2 \\
Subglottic oedema or stenosis from prolonged & 2 \\
intubation & 1 \\
Tracheostomy &
\end{tabular}

One patient had had a fundoplication, and did not have signs of reflux at aortopexy.
Correspondence to: Mr Kiely.

Accepted 8 November 1989
Hospital for Great Ormond Street, London WC1N 3JH

P S Malone 
Table 3 Extent of disease in 12 patients with tracheomalacia and five with tracheobronchomalacia

\begin{tabular}{ll}
\hline & $\begin{array}{l}\text { No of } \\
\text { patients }\end{array}$ \\
\hline Tracheomalacia: & \\
Upper third trachea & 0 \\
Middle third trachea & 2 \\
Lower third trachea & 8 \\
Lower two thirds trachea & 2 \\
Tracheobronchomalacia: & 2 \\
Lower two thirds trachea, both main bronchi & 2 \\
Lower two thirds trachea, left main bronchus & 1 \\
Lower third trachea, both main bronchi & 1 \\
Lower third trachea, right main bronchus & 1
\end{tabular}

attempts at decannulation had failed, and she still had an isolated segment of tracheomalacia.

The diagnosis was always confirmed by bronchoscopy. Complete or almost complete occlusion of the airway was seen during quiet respiration in each case. Specific radiological views of the airways were also taken, but these were not helpful. The radiological investigation of most value was dynamic screening of the airways. Using the information provided by these investigations, the extent of tracheomalacia or tracheobronchomalacia could be defined (table 3).

Aortopexy was performed as previously described. $^{3} 5$

\section{Results}

There were no early deaths. One patient had a respiratory arrest within hours of operation and required intubation and ventilation for a short period. The most common postoperative complication was respiratory tract infection, and one patient developed a wound haematoma.

Six patients $(35 \%)$ were cured with permanent relief of symptoms, and one (6\%) was improved. Aortopexy failed to relieve symptoms in 10 patients (59\%). Two patients underwent repeat aortopexy and tracheopexy, but these were not successful. Six patients in whom aortopexy failed had severe gastro-oesophageal reflux, which was confirmed by subsequent investigation. Four had a Nissen fundoplication, three of whom improved, and one of two patients who were treated medically also improved. This left six of the initial 17 patients in whom there was no symptomatic improvement. Four required tracheostomy, and only two have been successfully decannulated (one requiring a rib graft). One is awaiting decannulation and a patient with cerebral palsy who had a tracheostomy died from repeated chest infections at his local hospital. The patient who had a tracheostomy before aortopexy was not decannulated two years later because she was lost to follow up. Symptoms in the remaining patient were not severe enough to require tracheostomy but he had needed repeated hospital admissions for recurrent chest infections. Tracheobronchomalacia was not associated with failure; three of the five patients improved after aortopexy, with or without an additional Nissen fundoplication.

\section{Discussion}

Our results after aortopexy in this series con- trast with our previously reported success in patients with tracheomalacia associated with oesophageal atresia and tracheo-oesophageal fistulas. ${ }^{3}$ In that series 19 of 22 patients $(86 \%)$ were considerably improved after aortopexy, but only seven of 17 patients $(41 \%)$ benefited in the present study. Three of the five patients with tracheobronchomalacia improved, suggesting that the inclusion of these patients was not the cause of the disappointing results. The 12 patients with tracheomalacia had disease in an isolated segment and no patient had a completely diseased intrathoracic trachea, a feature known to have a poor prognosis. ${ }^{12}$ It is, therefore, difficult to explain the failure of aortopexy in primary tracheomalacia.

We emphasise the association between primary tracheomalacia and gastro-oesophageal reflux, particularly in those patients for whom aortopexy failed. Blair et al reported similar findings in 1986, and we agree that it is sometimes difficult to determine whether it is the tracheomalacia or the reflux that is responsible for the clinical picture. ${ }^{2}$ At present we rely on our bronchoscopic findings when deciding which condition takes priority, but it is possible in the future that the finding of reflux during sleep on 24 hour $\mathrm{pH}$ monitoring will identify this as the principle disease, and thus influence treatment. ${ }^{6}$ As our results now suggest that both conditions may need treatment, however, we advocate aggressive investigation and treatment of both the gastro-oesophageal reflux and the tracheomalacia.

As tracheomalacia is a self limiting condition that usually resolves spontaneously as the trachea stiffens, less aggressive forms of treatment have been advocated, including prolonged intubation and continuous positive airway pressure. $^{78}$ The main disadvantage of this is that patients must stay in hospital for periods of a year or more. Prolonged intubation may cause subglottic oedema or stenosis (as occurred in two of our patients), a complication that has its own morbidity and mortality. The application of continuous positive airway pressure by nasopharyngeal prong also requires care in hospital, and we have found in a limited number of patients that it does not reliably prevent airway collapse. We therefore believe that some more definitive form of treatment is required.

Tracheostomy should be avoided as a primary procedure and used only as a last resort. ${ }^{19}$ It can cause secondary tracheomalacia, tracheal fibrosis, and formation of granulation tissue. In this study five patients had tracheostomies, but only one was carried out as a primary procedure (at another hospital). The other four were undertaken after aortopexy had failed. One of these patients (who had severe cerebral palsy) died when continued treatment was not considered justified. Of the remaining three patients, two have been decannulated and are alive and well, and one is awaiting decannulation. The patient with the primary tracheostomy who was lost to follow up after aortopexy is only now being assessed for decannulation.

Based on this experience our recommendations are as follows. All patients with suspected tracheomalacia should have the diagnosis con- 
firmed by bronchoscopy. Gastro-oesophageal reflux should always be sought by barium studies, 24 hour $\mathrm{pH}$ monitoring, and oesophagoscopy. At the present time we recommend early aortopexy for those with severe symptoms, but in the future-with the widespread use of 24 hour pH monitoring-it may be possible to select those patients who would benefit from antireflux treatment alone. In patients with gastro-oesophageal reflux in whom aortopexy fails a Nissen fundoplication should be undertaken without delay. If this combined approach fails, tracheostomy is required.

We thank $\mathrm{Mr} \mathrm{CM}$ Bailey, consultant ear, nose, and throat surgeon, and $\operatorname{Dr} \mathrm{R}$ Dinwiddie and Dr D Matthew, consultant paediatricians, for referring these patients.
1 Greenholz SK, Karrer FM, Lilly JR. Contemporary surgery of tracheomalacia. I Pediatr Surg 1986;21:511-4.

2 Blair SK, Cohen R, Filler RM. Treatment of tracheomalacia: eight years' experience. F Pediatr Surg 1987;21:781-5.

3 Kiely EM, Spitz L, Brereton R. Management of tracheomalacia by aortopexy. Pediatric Surgery International 1987;2: $13-5$.

4 Vinograd I, Filler RM, Bahoric A. Longterm functional results of prosthetic airway splinting in tracheomalacia and results of prosthetic airway splinting in tracheom
bronchomalacia. I Pediatr Surg 1987;22:38-41.

5 Kiely EM. Aortopexy. In: Spitz L, Nixon HH, eds. Rob and Smith's operative surgery: paediatric surgery. 4th ed. London: Butterworths, 1988:126-9.

6 Newman LJ, Russe J, Glassman MS, et al. Patterns of gastrooesophageal reflux (GER) in patients with apparent lifethreatening events. I Pediatr Gastroenterol Nutr 1989; 8:157-60.

7 Pizer BL, Freeland AP, Wilkinson AR. Prolonged positive airway pressure for severe neonatal tracheobronchomalacia. Arch Dis Child 1986;61:908-9.

8 Sotomayor JL, Godinez PRI, Borden S, et al. Large airway collapse due to acquired tracheobronchomalacia in infancy. Am $\mathcal{J}$ Dis Child 1986;140:367-71.

9 Wailoo MP, Emery JL. The trachea in children with tracheooesophageal fistula. Histology 1979;3:329-38. 